\title{
NOTICE
}

\section{MATHEMATICAL PROGRAMMING STUDY 31 Computational Mathematical Programming}

\author{
Edited by K.L. Hoffman, R.H.F. Jackson and J. Telgen
}

This study is an outgrowth of the NATO Advanced Study Institute on Computational Mathematical Programming that was held in Bad Windsheim, FRG, July 23, 1984. The papers in this Study were drawn from, but not limited to, those contributed papers that appeared at the conference. The conference was sponsored by the Committee On Algorithms (COAL) of the Mathematical Programming Society. The director of the conference was Klaus Schittkowski, and co-directors were Karla Hoffman and Jan Telgen. The invited tutorials of that conference appear in a companion ASI publication entitled Computational Mathematical Programming (1985) edited by Klaus Schittkowski, and published by Springer-Verlag.

The general purpose of this conference and these two texts is to highlight recent research designed to predict the performance of mathematical programming software. These papers and talks are not concerned primarily with the description of a new algorithm but rather with the process that continues through the production of quality software. When we speak of "computational mathematical programming" we mean the sequence of research whereby algorithms are transformed into software which has been tested experimentally. This testing requires the searching for suitable test problems, and the executing of a designed experiment to determine the efficiency, robustness, reliability and applicability of various algorithms.

The papers presented in this study deal with many different aspects of computational mathematical programming. Some papers describe the testing of new algorithms for optimizing functions of a specified form while others present computational comparisons of known algorithms.

Taken as a whole, this collection of papers reflects both the current technology available to mathematical programmers for solving optimization problems and our mechanisms for testing and determining the quality of the software used. Abstracts of the papers follow.

Faiz A. Al-Khayyal, An implicit enumeration procedure for the general linear complementarity problem

An algorithm is presented for solving a quadratic programming formulation of the linear complementarity problem. No assumptions on the problem data are required. The algorithm is 
designed to solve the problem by implicitly enumerating the $2^{n}$ complementary cones. Geometrically, the procedure amounts to searching the extreme points of a sequence of faces of the constraint polyhedron of decreasing dimension. An extension to the procedure for finding all solutions of an arbitrary complementarity problem is also discussed.

The procedure has been implemented and tested on forty randomly generated problems (up to fifty dimensional) having dense indefinite defining matrices. The results of these tests demonstrate the superiority of this approach over two competing methods.

\section{M.C. Bartholomew-Biggs, Recursive quadratic programming methods based on the augmented Lagrangian}

This paper describes a method for constrained optimization which obtains its search directions from a quadratic programming subproblem based on the well-known augmented Lagrangian function. The method can be viewed as a development of the algorithm REQP which is related to the classical exterior point penalty function: and it is argued that the new technique will have certain computational advantages arising from the fact that it need not involve a sequence of penalty parameters tending to zero. An algorithm with global convergence for equality constrained problems is presented. Computational results are also given for this algorithm and some alternative strategies are briefly considered for extending it to deal with inequality constraints.

\section{Ron S. Dembo, A primal truncated Newton algorithm with application to large-scale nonlinear network optimization}

We describe a new, convergent, primal-feasible algorithm for linearly constrained optimization. It is capable of rapid asymptotic behavior and has relatively low storage requirements. Its application to large-scale nonlinear network optimization is discussed and computational results on problems of over 2000 variables and 1000 constraints are presented. Indications are that it could prove to be significantly better than known methods for this class of problems.

\section{Sjur D. Flåm, Approximating some convex programs in terms of Borel fields}

The paper considers approximations of convex programs involving expectation functionals. These approximations are based on conditional expectations and yield variational convergence for all relevant topologies on the decision space.

\section{Harvey J. Greenberg, Computer-assisted analysis for diagnosing infeasible or unbounded linear programs}

This paper presents methods to provide computer-assisted analysis to diagnose infeasible or unbounded linear programs. This may be for retrospective analysis, after the difficulty has been detected, or for preventive analysis. The first method is by successive reduction of bounds on primal quantities and dual prices. This is the most commonly used method in practice due to the availability of REDUCE procedures in commercial mathematical programming systems. The second method is by path or cycle generation, introduced by the author in a more general context of solution analysis. The third method is by "netforming," and the fourth is by coefficient screening. The last two methods are untried in practice, but it is suggested how they may provide a useful aid, particularly in certain computing environments. Collectively, these four methods comprise a foundation for further research. 
D.W. Hearn, S. Lawphongpanich and J.A. Ventura, Restricted simplicial decomposition: Computation and extensions

Restricted simplicial decomposition (RSD) is a very useful technique for certain large-scale pseudoconvex programming problems such as the traffic assignment problem and other network flow problems. The "restricted" version of this method allows the user to treat the maximum size of the generated simplices as a parameter. When the parameter is at its minimum value, the method reduces to the Frank-Wolfe algorithm; when at its maximum, the original simplicial decomposition of von Hohenbalken and Holloway results. Computer storage requirements increase linearly with the parameter, but our computational experiments on a variety of test problems show that there is a payoff in improved progress of the method as measured by the relative error in the objective function. Included in the tests are a number of real-world traffic networks, some large electrical networks, a water distribution network, and linearly constrained problems of the Colville study.

Conditions are given for which RSD converges after a finite number of major cycles, and variations of RSD which have potential for real-world applications are developed. These include a quadratic approximation of the master problem and an extension to include the case of unbounded subproblems.

James K. Ho, Recent advances in the decomposition approach to linear programming

Recent development and experience with real applications of the decomposition approach to solving large structured linear programs indicate that its potential advantages that are so appealing conceptually and yet so elusive in forthcoming may eventually be realized. New theoretical efforts to improve the robustness of the approach as well as to exploit parallel computation are also on the horizon.

\section{Michael Kupferschmid and J.G. Ecker, A note on solution of nonlinear programming problems with imprecise function and gradient values}

A model for introducing imprecisions in function and gradient values is developed and computational results are presented showing how the performance of some nonlinear programming algorithms degrades as the level of precision in the function and gradient values decreases.

\section{Z.A. Maany, A new algorithm for highly curved constrained optimisation}

This paper describes a new algorithm for highly curved constrained optimisation. The algorithm under discussion makes use of the second derivatives of both the objective function and constraints. At every iteration a subproblem based on the second order approximation of the objective and constraints functions is solved. Three strategies to solve the subproblem are used. Some computational results are given. Although the performance of the subroutine is very promising a number of areas are still under development and further improvement is expected.

\section{Robert Mifflin, An implementation of an algorithm for univariate minimization and an application to nested optimization}

This paper provides an efficient numerical algorithm for solving quite general single variable constrained minimization problems. Also given are a listing of the corresponding FORTRAN 
subroutine PQ1, an explanation of its general use and a description of its particular use in solving a practical resource allocation problem via a dual technique requiring nested optimization.

\section{Włodzimierz Ogryczak, On practical stopping rules for the simplex method}

Well defined feasibility tolerances are necessary to guarantee reliable results for the simplex algorithm. This note presents some formulae for dynamic definition of suitable values of the tolerances at each simplex step. They are based on error analysis techniques and seem to be applicable in the standard simplex codes. The tolerances proved to be useful in practice when lexicographic LP problems were solved.

\section{J.A. Tomlin, An experimental approach to Karmarkar's projective method for linear programming}

This paper describes the evolution of an experimental implementation of the Karmarkar projective method and gives computational results for some small to medium, but realistically structured models. 